\title{
Erratum: Acetate-mediated novel survival strategy against drought in plants
}

Jong-Myong Kim, Taiko Kim To, Akihiro Matsui, Keitaro Tanoi, Natsuko I. Kobayashi, Fumio Matsuda, Yoshiki Habu, Daisuke Ogawa, Takuya Sakamoto, Sachihiro Matsunaga, Khurram Bashir, Sultana Rasheed, Marina Ando, Hiroko Takeda, Kanako Kawaura, Miyako Kusano, Atsushi Fukushima, Takaho A. Endo, Takashi Kuromori, Junko Ishida, Taeko Morosawa, Maho Tanaka, Chieko Torii, Yumiko Takebayashi, Hitoshi Sakakibara, Yasunari Ogihara, Kazuki Saito, Kazuo Shinozaki, Alessandra Devoto and Motoaki Seki

Nature Plants 3, 17097 (2017); published 26 June 2017; corrected 17 July 2017.

The Supplementary Information files originally published with this Letter were not the intended final versions. The Supplementary Information PDF and Supplementary Tables 3, 5 and 6 have now been updated to fix a number of cosmetic problems. No data have been affected and there have been no changes in meaning. 